\title{
Dark photon decay beyond the Euler-Heisenberg limit
}

\author{
Samuel D. McDermott, ${ }^{1}$ Hiren H. Patel, ${ }^{2}$ and Harikrishnan Ramani ${ }^{1}$ \\ ${ }^{1}$ C. N. Yang Institute for Theoretical Physics, Stony Brook, New York 11794, USA \\ ${ }^{2}$ Amherst Center for Fundamental Interactions, Department of Physics, University of Massachusetts, \\ Amherst, Massachusetts 01003, USA
}

(Received 24 May 2017; published 23 April 2018)

\begin{abstract}
We calculate the exact width for a dark photon decaying to three photons at one-loop order for dark photon masses $m^{\prime}$ below the $e^{+} e^{-}$production threshold of $2 m_{e}$. We find substantial deviations from previous results derived from the lowest order Euler-Heisenberg effective Lagrangian in the range $m_{e} \lesssim m^{\prime} \leq 2 m_{e}$, where higher order terms in the derivative expansion are non-negligible. This mass range is precisely where the three photon decay takes place on cosmologically relevant timescales. Our improved analysis reveals a window for dark photons in the range $850 \mathrm{KeV} \lesssim m^{\prime} \leq 2 m_{e}, 10^{-5} \lesssim \epsilon \lesssim 10^{-4}$ that is only constrained by possibly model-dependent bounds on the number of light degrees of freedom in the early Universe.
\end{abstract}

DOI: 10.1103/PhysRevD.97.073005

\section{INTRODUCTION}

Matter with no standard model gauge charges may be charged under an Abelian gauge group of its own. The corresponding "dark photon" is an ingredient of many compelling extensions to the standard model $[1,2]$. A new gauge boson of this type may kinetically mix with the standard model photon [3] via a gauge invariant dimensionfour operator $\mathcal{O}_{\text {mix }}=-\epsilon F_{\mu \nu}^{\prime} F^{\mu \nu} / 2$, where $F^{\prime}$ is the field strength of the dark photon $A^{\prime}$ and $F$ is the field strength of the standard model photon $A$. This operator induces a coupling of the dark photon with the electromagnetic current

$$
\mathcal{L}=\epsilon A_{\mu}^{\prime} J_{\mathrm{EM}}^{\mu}
$$

proportional to the kinetic mixing parameter $\epsilon$. Most searches for the dark photon rely on the observation of the products of its decay after it has been produced in lowenergy terrestrial experiments [4-16], although some search strategies are sensitive even to invisibly decaying or effectively stable dark photons [17-21].

We are interested in the visible decays of low-mass dark photons. Below the $e^{+} e^{-}$threshold, the dominant decay channel is $A^{\prime} \rightarrow \gamma \gamma \gamma$. [The weak decay $A^{\prime} \rightarrow \nu \bar{\nu}$ is effectively suppressed by $\left(\mathrm{m}^{\prime} / m_{W^{ \pm}}\right)^{4} \sim 10^{-21}\left(\mathrm{~m}^{\prime} / m_{e}\right)^{4}$, while decay to two photons is forbidden by the Landau-Yang theorem.] The three photon decay width was previously

Published by the American Physical Society under the terms of the Creative Commons Attribution 4.0 International license. Further distribution of this work must maintain attribution to the author(s) and the published article's title, journal citation, and DOI. Funded by SCOAP ${ }^{3}$. estimated from the Euler-Heisenberg effective action [22], which we discuss below. But since the Euler-Heisenberg effective action provides only the leading term in the derivative expansion with a small parameter given by $k_{\gamma}^{2} / m_{e}^{2}$, the derived decay width is expected to be valid provided $m^{\prime} \ll m_{e}$. For characteristic energies close to the electron mass, as expected for a decaying dark photon of mass in the range $m_{e} \lesssim m^{\prime} \lesssim 2 m_{e}$, this approximation breaks down, requiring a more exact treatment.

In this work, we improve the calculation for the $A^{\prime} \rightarrow \gamma \gamma \gamma$ decay width by evaluating it in quantum electrodynamics at one-loop order exactly, obtaining a result valid for all dark photon masses below the $e^{+} e^{-}$threshold. We determine that the exact width is up to 2 orders of magnitude larger than the leading-order result. We investigate the implications for a dark photon that comprises a non-negligible cosmological density in the early Universe. Interestingly, a small region in parameter space at $10^{-5} \lesssim \epsilon \lesssim 10^{-4}$ and $850 \mathrm{KeV} \lesssim m^{\prime} \leq 2 m_{e}$ is reopened: a dark photon with these properties is allowed by all model-independent considerations.

\section{CORRECTIONS TO EULER-HEISENBERG LAGRANGIAN(S)}

The Euler-Heisenberg Lagrangian is the low-energy effective Lagrangian that is matched to quantum electrodynamics at energies below the electron mass. This Lagrangian predicts gauge boson self-couplings that do not exist at higher energies, leading to photon self-interactions [23]. In general, we may construct an Euler-Heisenberg-like Lagrangian for any (product of) gauge theories after the lightest charged particles have been integrated out. Gauge bosons with bifundamental matter acquire any contact 


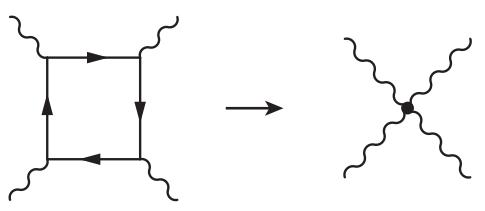

FIG. 1. The dynamics of an Abelian gauge theory below all charged particle masses includes gauge boson self-scattering. At leading order, this is governed by the Euler-Heisenberg Lagrangian.

interactions that are the remnant of charge-conserving interactions of box diagrams of heavy matter, as shown in Fig. 1.

The Euler-Heisenberg Lagrangian is a nonperturbative object that predicts interactions of arbitrarily many (even) numbers of photons order by order in perturbation theory. In four dimensions, the term involving four photons is [24]

$$
\mathcal{L}_{\mathrm{EH}, 4 \gamma}=\frac{\alpha^{2}}{90 m^{4}}\left[\left(F_{\mu \nu} F^{\mu \nu}\right)^{2}-\frac{7}{4}\left(F_{\mu \nu} \tilde{F}^{\mu \nu}\right)^{2}\right]
$$

where $m$ is the charged particle mass. (Henceforth we specialize to the QED case, with an electron in the loop, such that $m \rightarrow m_{e}$.) The Lagrangian in Eq. (2) famously allows the computation of a cross section for light-by-light scattering [25]. Replacing one of the four photons with a dark photon and inserting a factor $\epsilon$ for the electron coupling to the dark photon gives us the Lagrangian for the process that we are interested in. Accounting for the reduced symmetry, we find that Eq. (2) generalizes to

$\mathcal{L}_{\mathrm{EH}, \mathrm{dark}}=\frac{\epsilon \alpha^{2}}{45 m_{e}^{4}}\left(14 F_{\mu \nu}^{\prime} F^{\nu \lambda} F_{\lambda \rho} F^{\rho \mu}-5 F_{\mu \nu}^{\prime} F^{\mu \nu} F_{\alpha \beta} F^{\alpha \beta}\right)$,

in agreement with [22].

However, the Lagrangians in Eqs. (2) and (3) do not produce the full gauge boson scattering cross sections for arbitrary energies. This is well known for QED: the cross section for light-by-light scattering was obtained by Karplus and Neuman [26] and these "nonlinearities" were fully explored by de Tollis and collaborators [27]. There, they reproduced the $m_{e}^{-8}$ scaling predicted by the EulerHeisenberg Lagrangian at small energy transfer, but found that this deviated from the full result close to the electron mass.

The reason the Euler-Heisenberg Lagrangian fails close to the electron mass is because it is the first term in an expansion in derivatives on the field strength. Although the Euler-Heisenberg Lagrangian is a nonperturbative object that contains infinitely many terms, each term includes $2 n$ products of field strength tensors, for integer $n \geq 2$, with $4(n-1)$ powers of $m_{e}$ in the denominator of each term. No additional terms with more derivatives appear in this expansion. Thus, Lagrangians obtained from the Euler-Heisenberg Lagrangian, such as those in Eqs. (2) and (3) as well as those with additional photon fields, are only the leading terms of the $2 n$ photon interaction in the $m_{e} \rightarrow \infty$ limit. Each of these terms receives $\mathcal{O}(1)$ corrections when the components of $\partial_{\alpha} F_{\mu \nu} / m_{e}$ are comparable to those of $F_{\mu \nu}$. Higher derivative terms fill out the effective theory at energies comparable to $m_{e}$. Infinitely many derivatives are needed to get the exact result, indicating a nonlocality at small distances.

Of course, the full theory that completes the EulerHeisenberg Lagrangian is known, and this nonlocality is resolved by $m_{e}$. For four-photon interactions, the first two subleading Lagrangian terms containing two and four derivatives have in fact been obtained by a matching calculation using the full QED scattering cross section [28]. By calculating in QED, the authors of [28] found that corrections to light-by-light scattering are an order of magnitude when each incoming photon energy equals $m_{e}$. The corrections to the three photon decay are naïvely similar to the corrections for light-by-light scattering, and our exact calculations described below reveal that the corrections in this context can be even larger.

\section{DARK PHOTON DECAY}

We now calculate the dark photon partial width $A^{\prime}\left(k^{\prime}\right) \rightarrow$ $\gamma\left(k_{1}\right) \gamma\left(k_{2}\right) \gamma\left(k_{3}\right)$ exactly at one-loop order in quantum electrodynamics. Three of the six diagrams contributing to the decay amplitude are shown in Fig. 2. By charge conjugation invariance, the other three diagrams with opposite electron flow are equal in value to these diagrams. Denoting the amplitude as

$$
i \mathcal{M}=i \mathcal{A}^{\mu \nu \rho \sigma} \epsilon\left(k^{\prime}\right)_{\mu} \epsilon^{*}\left(k_{1}\right)_{\nu} \epsilon^{*}\left(k_{2}\right)_{\rho} \epsilon^{*}\left(k_{3}\right)_{\sigma},
$$

where the $\epsilon\left(k_{i}\right)$ are the polarization four-vectors of the external photons, the Feynman amplitude for the dark photon decay derived from Eq. (1) is

$$
\begin{aligned}
& i \mathcal{A}^{\mu \nu \rho \sigma}=-2 \epsilon e^{4}\left[\int \frac{d^{4} p}{(2 \pi)^{4}} \frac{\operatorname{Tr}\left[\gamma^{\mu}\left(\not p+m_{e}\right) \gamma^{\nu}\left(\not p+\not \not_{1}+m_{e}\right) \gamma^{\rho}\left(\not p+\not k_{1}+\not k_{2}+m_{e}\right) \gamma^{\sigma}\left(\not p+\not \not^{\prime \prime}+m_{e}\right)\right]}{\left[p^{2}-m_{e}^{2}\right]\left[\left(p+k_{1}\right)^{2}-m_{e}^{2}\right]\left[\left(p+k_{1}+k_{2}\right)^{2}-m_{e}^{2}\right]\left[\left(p+k^{\prime}\right)^{2}-m_{e}^{2}\right]}\right. \\
& +\int \frac{d^{4} p}{(2 \pi)^{4}} \frac{\operatorname{Tr}\left[\gamma^{\mu}\left(\not p+m_{e}\right) \gamma^{\rho}\left(\not p+\not k_{2}+m_{e}\right) \gamma^{\nu}\left(\not \not+\not k_{1}+\not k_{2}+m_{e}\right) \gamma^{\sigma}\left(\not p+\not k^{\prime}+m_{e}\right)\right]}{\left[p^{2}-m_{e}^{2}\right]\left[\left(p+k_{2}\right)^{2}-m_{e}^{2}\right]\left[\left(p+k_{1}+k_{2}\right)^{2}-m_{e}^{2}\right]\left[\left(p+k^{\prime}\right)^{2}-m_{e}^{2}\right]} \\
& \left.+\int \frac{d^{4} p}{(2 \pi)^{4}} \frac{\operatorname{Tr}\left[\gamma^{\mu}\left(\not p+m_{e}\right) \gamma^{\nu}\left(\not p+\not k_{1}+m_{e}\right) \gamma^{\sigma}\left(\not p+\not k^{\prime}-\not k_{2}+m_{e}\right) \gamma^{\rho}\left(\not p+\not k^{\prime}+m_{e}\right)\right]}{\left[p^{2}-m_{e}^{2}\right]\left[\left(p+k_{1}\right)^{2}-m_{e}^{2}\right]\left[\left(p+k^{\prime}-k_{2}\right)^{2}-m_{e}^{2}\right]\left[\left(p+k^{\prime}\right)^{2}-m_{e}^{2}\right]}\right] \text {. }
\end{aligned}
$$




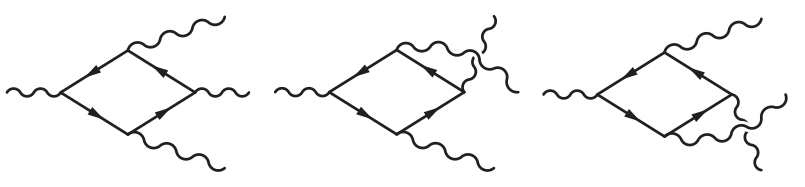

FIG. 2. Three of six Feynman diagrams contributing to the dark photon decay amplitude at one-loop order. The particle in the loop is an electron. The three other diagrams are obtained by reversing the electron flow.

We evaluate the amplitude and decay width using the algebraic reduction method of Passarino and Veltman [29] implemented in PACKAGE-X [30]. We have checked that the total amplitude is free of ultraviolet divergences and that the amplitude is transverse to all external momenta

$$
k_{\mu}^{\prime} \mathcal{A}^{\mu \nu \rho \sigma}=k_{1 \nu} \mathcal{A}^{\mu \nu \rho \sigma}=k_{2 \rho} \mathcal{A}^{\mu \nu \rho \sigma}=k_{3 \sigma} \mathcal{A}^{\mu \nu \rho \sigma}=0,
$$

as required by the Abelian Ward identity. After the algebraic reduction to scalar functions, we square the amplitude, sum over final photon polarizations, and average over the initial dark photon polarization using the completeness relation valid for transverse amplitudes

$$
\begin{aligned}
{\overline{|\mathcal{M}|^{2}}=}^{2} & \frac{1}{3} \mathcal{A}^{\mu \nu \rho \sigma}\left(\mathcal{A}^{*}\right)^{\dot{\mu} \dot{\nu} \dot{\rho} \dot{\sigma}} \\
& \times\left(-g_{\mu \dot{\mu}}+\frac{k_{\mu}^{\prime} k_{\dot{\mu}}^{\prime}}{m^{\prime 2}}\right)\left(-g_{\nu \dot{\nu}}\right)\left(-g_{\rho \dot{\rho}}\right)\left(-g_{\sigma \dot{\sigma}}\right) \\
= & \frac{1}{3} \mathcal{A}^{\mu \nu \rho \sigma}\left(\mathcal{A}^{*}\right)_{\mu \nu \rho \sigma} .
\end{aligned}
$$

We obtain the dark photon decay width by integrating over the three body phase space in terms of the Dalitz variables $m_{12}^{2}=\left(k_{1}+k_{2}\right)^{2}$ and $m_{13}^{2}=\left(k_{1}+k_{3}\right)^{2}$,

$\Gamma_{\gamma \gamma \gamma}=\frac{1}{(2 \pi)^{3}} \frac{1}{32 m^{13}} \frac{1}{6} \int_{0}^{m^{\prime 2}} d m_{12}^{2} \int_{0}^{m^{\prime 2}-m_{12}^{2}} d m_{13}^{2} \overline{|\mathcal{M}|^{2}}$,

where the factor of $1 / 6$ accounts for identical final state particles.

There are two potential sources of numerical instability in the numerical evaluation of the decay amplitude. If the dark photon mass is much smaller than the electron mass $m^{\prime 2} \ll m_{e}^{2}$, or on the boundary of the phase space (where a final state invariant mass becomes small compared to the electron mass, e.g. $m_{12}^{2}, m_{13}^{2}$ or $m_{23}^{2} \ll m_{e}^{2}$ ) the kinematic Gram determinant becomes small and the PassarinoVeltman reduction formula becomes invalid. We circumvent this problem in PACKAGE- $X$ by evaluating the amplitudes with sufficient working precision to achieve a numerically stable result throughout the entire physical region. With enough runtime, we can attain any desired precision even arbitrarily close to $m^{\prime}=2 m_{e}$. Other physical effects like mixing with the orthopositronium resonance and higher order Coulomb effects may begin

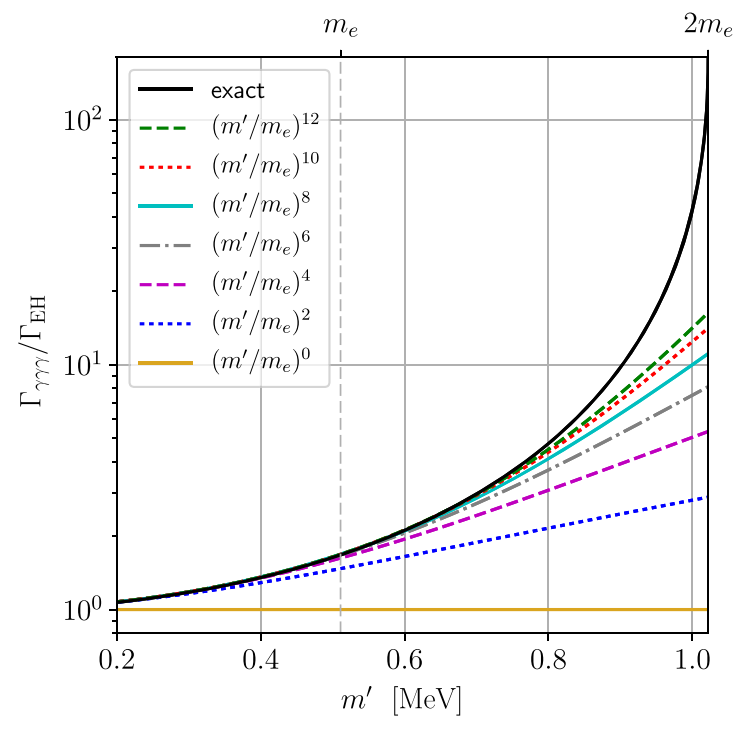

FIG. 3. Dark photon decay width to three photons in units of the width obtained in the Euler-Heisenberg limit. We show corrections including terms up to sixth order in the variable $m^{\prime 2} / m_{e}^{2}$ as well as the exact correction (in black).

to be non-negligible in this regime, and though the former are expected to be small [31] both effects are worthy of investigation in future work. We show the exact evaluation of the decay width in Fig. 3. We provide a supplemental file [32] containing the data points we used to generate the black line. Additionally, a program that calculates the differential width as a Mathematica program (requiring PACKAGE-X) or as a Fortran program (requiring the COLLIER [33] library) may be requested from the authors. Exact results may in principle be extracted from [34].

By differentiating the Passarino-Veltman functions arising in the covariant decomposition of the amplitude in Eq. (5), we may also obtain an analytic representation of the partial width as a series in inverse electron mass,

$$
\Gamma_{\gamma \gamma \gamma}=\Gamma_{\mathrm{EH}}\left[1+\sum_{k=1}^{\infty} c_{k}\left(\frac{m^{2}}{m_{e}^{2}}\right)^{k}\right]
$$

which we expect to converge out to the onset of the physical threshold at $\mathrm{m}^{\prime 2} / \mathrm{m}_{e}^{2}=4$. We confirm that the leading term $\Gamma_{\mathrm{EH}}$ is determined by the amplitude in the Euler-Heisenberg limit derived from Eq. (3),

$\Gamma_{\mathrm{EH}}=\frac{17 \epsilon^{2} \alpha_{\mathrm{EM}}^{4}}{11664000 \pi^{3}} \frac{m^{\prime 9}}{m_{e}^{8}} \simeq 1 \mathrm{~s}^{-1}\left(\frac{\epsilon}{0.003}\right)^{2}\left(\frac{m^{\prime}}{m_{e}}\right)^{9}$,

in agreement with [22]. In the context of the EulerHeisenberg effective theory discussed in the previous section, the remaining terms in Eq. (9) arise from corrections to the Euler-Heisenberg effective action involving derivatives of the field strength tensors. We give the first six 
TABLE I. Series coefficients of the large electron mass expansion of the dark photon decay $A^{\prime} \rightarrow \gamma \gamma \gamma$ given in Eq. (9). The final column represents the correction to the decay width due to each term at the threshold value of $m^{\prime 2} / m_{e}^{2}=4$ relative to the Euler-Heisenberg estimate Eq. (10).

\begin{tabular}{lcc}
\hline \hline & $c_{k}$ & $c_{k} \times 4^{k}$ \\
\hline$c_{1}$ & $\frac{335}{714}$ & 1.88 \\
$c_{2}$ & $\frac{128941}{839664}$ & 2.46 \\
$c_{3}$ & $\frac{44787}{1026256}$ & 2.79 \\
$c_{4}$ & $\frac{1249649333}{108064756800}$ & 2.96 \\
$c_{5}$ & $\frac{36494147}{12382420050}$ & 3.02 \\
$c_{6}$ & $\frac{867635449}{1614300688000}$ & 2.20 \\
\hline \hline
\end{tabular}

coefficients $c_{k}$ in Table I, and in Fig. 3 we plot the corresponding corrections to the partial width.

As the dark photon mass approaches the electron pair production threshold, each term supplies a correction that is individually larger than the width in the Euler-Heisenberg limit. We demonstrate this in the third column of Table I. Thus, although the sixth order correction gives an acceptable approximation to the exact width up to $m^{\prime} \simeq 800 \mathrm{KeV}$, as shown in Fig. 3, asymptotically many terms are required for an accurate estimate of the partial width for $m^{\prime} \simeq 2 m_{e}$. At threshold, we find that the dark photon partial width is over 2 orders of magnitude larger than the width in the Euler-Heisenberg limit. This means that the dark photon lifetime is drastically shorter in this mass range than previously appreciated. This has important phenomenological consequences, which we discuss next.

\section{UPDATED CONSTRAINTS ON DARK PHOTON MASS BELOW $2 m_{e}$}

A dark photon with kinetic mixing parameter larger than around $\epsilon \gtrsim 10^{-12}$ can be in thermal equilibrium at some point in the early Universe, and can thus play the role of cosmological dark matter or otherwise affect the evolution of the early Universe $[18,19]$. If the dark photon mass satisfies $m^{\prime} \leq 2 m_{e}$, the dark photon is quite long lived because the decay to three photons is loop mediated, contains four factors of $\alpha_{\mathrm{EM}}$, and must go into three body phase space, as in Eqs. (8) and (10). Thus, dark photons with mass $m^{\prime} \leq 2 m_{e}$ generically have long lifetimes, modulated by the kinetic mixing factor $\epsilon$. The decay to three photons can happen late enough to alter standard model physics in epochs where the thermal history of the Universe is well established. In this section, we describe the effects of such a long-lived dark photon, and we detail the bounds from considerations of this kind of latedecaying particle.

Roughly speaking, dark photon decays should not occur between the time of heavy element nucleosynthesis (BBN) and the formation of the cosmic microwave background

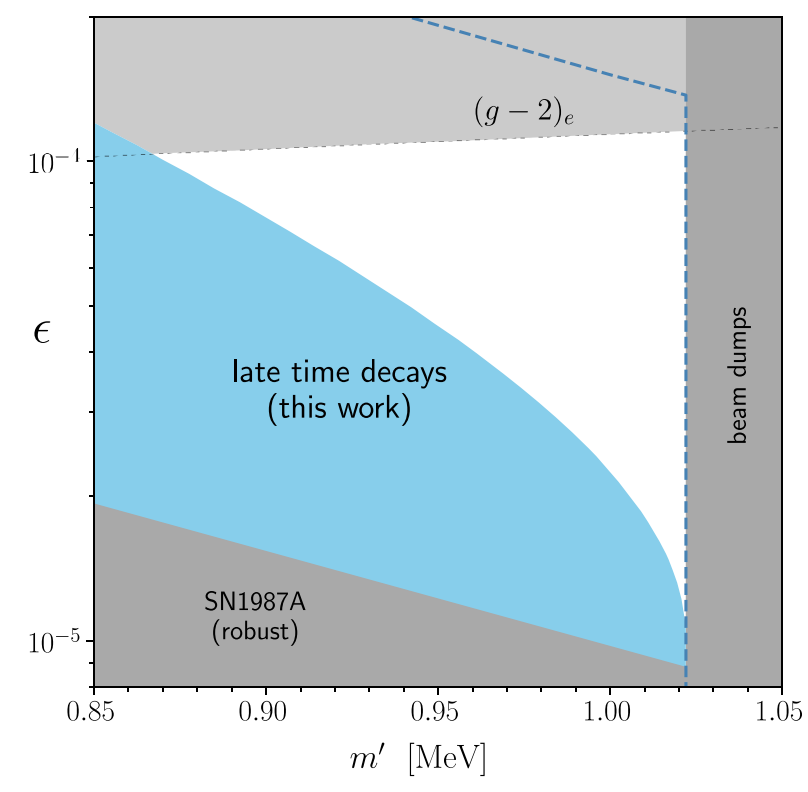

FIG. 4. Constraints on the dark photon parameter space. In the blue region the lifetime of the dark photon to decay to three photons exceeds $1 \mathrm{~s}$. There is an open window near $10^{-5} \lesssim \epsilon \lesssim$ $10^{-4}$ and $850 \mathrm{KeV} \lesssim m^{\prime} \lesssim 1 \mathrm{MeV}$. We also show constraints from SN1987A using the "robust" results from [21], constraints from the anomalous magnetic moment of the electron [35], constraints from beam dump experiments [1], and the results from the lifetime only using the leading-order Euler-Heisenberglike width (dashed blue).

(CMB) radiation, because the additional entropy injection could conflict with the required ratio of neutrons to protons during $\mathrm{BBN}$, which is set at a time of $1 \mathrm{~s}$, or the observed agreement between the baryon to photon ratio at $\mathrm{BBN}$ and the CMB. Decays from the time of formation of $\mathrm{CMB}$ through the cosmological dark ages are ruled out by limits on the $\mu$ and $y$ distortions of the CMB blackbody spectrum. Decays after the epoch of reionization until the present time are forbidden by limits on the amount of diffuse extragalactic background light. For $m^{\prime} \leq 2 m_{e}$, we thus conservatively rule out lifetimes

$$
1 \mathrm{~s} \leq 1 / \Gamma_{\gamma \gamma \gamma} \leq t_{\Phi}
$$

where $t_{\Phi}$ is a mass-dependent lifetime that is obtained from saturating observations of the high-latitude flux of x-ray photons, as in [36]. We illustrate the ramifications for the dark photon parameter space in Fig. 4.

We emphasize that there is some uncertainty on the lower bound in Eq. (11). Dark photons in this mass range are resonantly produced in the early Universe if the reheat temperature is sufficiently large compared to their mass, as shown in [18]. If the scattering process $e \gamma \leftrightarrow e A^{\prime}$ remains in equilibrium after this population is produced, as expected for the mixing angles we consider, the dark photon can remain abundant at $\mathrm{BBN}$, potentially with 
$n_{A^{\prime}} \gg n_{\gamma}$. However, at temperatures relevant for BBN, this rate is at least partially kinematically suppressed by the dark photon mass, and also the rate for $e \gamma \leftrightarrow e A^{\prime}$ is subject to plasma effects that suppress the mixing angle with the standard model photon. Conducting a thorough investigation of the cosmic evolution of this population (and hence the exact location of the allowed regions) for this range of masses and mixing angles is an interesting open problem that would require running Boltzmann codes for early universe abundances, akin to [37]. Thus, we restrict ourselves to simply updating earlier work that approximately rules out decays with $\tau \geq 1 \mathrm{~s}$; see e.g. [18].

This parameter space is possibly bounded by degree of freedom counting in the early Universe, which is logically independent from the decay bounds mentioned above. If dark photons remain in kinetic equilibrium until after the time of neutrino decoupling, as described above, they potentially add to the radiation budget of the Universe, thus speeding up the expansion rate and adversely affecting sensitive BBN processes. However, their decay to photons would increase the entropy of the standard model heat bath, leading to a net negative contribution to $N_{\text {eff }}$ [38]. Compensating contributions to the radiation budget could nevertheless come from slightly heavier weakly coupled particles [39], which plausibly exist in a complete model of the dark sector. This renders the counting of additional degrees of freedom model dependent for the scenario considered. Finding a UV-complete model necessary to avoid all of the bounds discussed here is a compelling, and nontrivial, goal for future work.

We point out an open window free of all constraints where $10^{-5} \lesssim \epsilon \lesssim 10^{-4}$ and $850 \mathrm{KeV} \lesssim m^{\prime} \leq 2 m_{e}$. This is precisely the mass range where subleading corrections to the Euler-Heisenberg-like Lagrangian are large. Our exact computation of the decay width is crucial to revealing this window: using the leading-order Euler-Heisenberg-like Lagrangian, one would conclude that dark photons of this mass have lifetimes longer than one second, leading to entropy injection after $\mathrm{BBN}$ and ruling out all mixing angles below $\epsilon \lesssim 0.003 \times\left(\mathrm{m}^{\prime} / m_{e}\right)^{-9 / 2}[21] .{ }^{1}$ Because the corrections near threshold that we have calculated indicate that the width is up to 2 orders of magnitude larger than the

\footnotetext{
${ }^{1}$ The possibility that the decay width from the Euler-Heisenberg Lagrangian might receive corrections near threshold was suggested in [21], although no estimate of their size was given.
}

Effective Field Theory result, we find that dark photons in this mass range decay well before BBN.

This window remains open because a dark photon in this window is weakly coupled enough that it does not affect the anomalous magnetic moment of the electron $[35,40]$ but strongly coupled enough that it is effectively trapped inside the protoneutron star during the detonation of Supernova 1987A: using the most conservative models of the core collapse process, in particular assuming a large progenitor mass and a stiff equation of state, we find that a dark photon in this parameter space does not carry out more energy than is released in standard model neutrinos [21]. About half of the parameter space is ruled out assuming a smaller progenitor mass and a mean-field neutrino transport model, however [21].

This range is potentially constrained by considerations of the SLAC millicharge experiment [41,42], which is sensitive to any light dark matter that can scatter off nuclei. In the near future, this window will also be subject to searches performed by new beam dump experiments that will be sensitive to any particle that can escape the beam as missing energy $[43,44]$. As a complementary technique, it is possible that improvements of the measurement of the anomalous magnetic moment of the electron will soon be sufficiently improved to constrain this window of parameter space [45]. Thus, this exciting window for new physics will be comprehensively and complementarily probed in a variety of upcoming experiments.

\section{ACKNOWLEDGMENTS}

We thank Zackaria Chacko, Rouven Essig, Marat Freytsis, Susan Gardner, and Jakub Scholtz for discussions. S. D. M. is supported by Grant No. NSF-PHY-1316617. H.R. is supported in part by NSF CAREER Grant No. NSF-PHY-1056833 and NSF Grant No. NSF-PHY1620628.

Note added.-Recently, two preprints [46,47] appeared suggesting that the region of parameter space that we indicate has been reopened is in fact ruled out by data from neutrino experiments. However, [46] strictly assumes $m^{\prime} \ll m_{e}$, which is inappropriate in this regime, and it is difficult to assess the exact signal process for Liquid scintillator neutrino detector in [47] (the Borexino-SOX experiment is not yet installed). We conclude that these preprints underline our assertion that this parameter space is probed by a wide variety of interesting experimental programs. 
[1] R. Essig et al., in Proceedings, 2013 Community Summer Study on the Future of U.S. Particle Physics: Snowmass on the Mississippi (CSS2013), Minneapolis, Minnesota, USA, 2013 (2013).

[2] J. Alexander et al., arXiv:1608.08632.

[3] B. Holdom, Phys. Lett. 166B, 196 (1986).

[4] B. Batell, M. Pospelov, and A. Ritz, Phys. Rev. D 79, 115008 (2009).

[5] R. Essig, P. Schuster, and N. Toro, Phys. Rev. D 80, 015003 (2009).

[6] M. Reece and L.-T. Wang, J. High Energy Phys. 07 (2009) 051 .

[7] F. Bossi, Phys. Rev. D 80, 015003 (2009).

[8] J. D. Bjorken, R. Essig, P. Schuster, and N. Toro, Phys. Rev. D 80, 075018 (2009).

[9] B. Batell, M. Pospelov, and A. Ritz, Phys. Rev. D 80, 095024 (2009).

[10] B. Aubert et al. (BABAR Collaboration), in Proceedings, 24th International Symposium on Lepton-Photon Interactions at High Energy (LP09), Hamburg, Germany, 2009 (2009).

[11] M. Freytsis, G. Ovanesyan, and J. Thaler, J. High Energy Phys. 01 (2010) 111.

[12] R. Essig, P. Schuster, N. Toro, and B. Wojtsekhowski, J. High Energy Phys. 02 (2011) 009.

[13] R. Essig, R. Harnik, J. Kaplan, and N. Toro, Phys. Rev. D 82, 113008 (2010).

[14] H. Merkel et al. (A1 Collaboration), Phys. Rev. Lett. 106, 251802 (2011).

[15] S. Abrahamyan et al. (APEX Collaboration), Phys. Rev. Lett. 107, 191804 (2011).

[16] F. Archilli et al. (KLOE-2 Collaboration), Phys. Lett. B 706, 251 (2012).

[17] B. Aubert et al. (BABAR Collaboration), in Proceedings, 34th International Conference on High Energy Physics (ICHEP 2008), Philadelphia, Pennsylvania, 2008 (2008).

[18] J. Redondo and M. Postma, J. Cosmol. Astropart. Phys. 02 (2009) 005.

[19] A. E. Nelson and J. Scholtz, Phys. Rev. D 84, 103501 (2011).

[20] H. An, M. Pospelov, and J. Pradler, Phys. Lett. B 725, 190 (2013).

[21] J. H. Chang, R. Essig, and S. D. McDermott, J. High Energy Phys. 01 (2017) 107.
[22] M. Pospelov, A. Ritz, and M. B. Voloshin, Phys. Rev. D 78, 115012 (2008).

[23] W. Heisenberg and H. Euler, Z. Phys. 98, 714 (1936).

[24] A. Ritz and R. Delbourgo, Int. J. Mod. Phys. A11, 253 (1996).

[25] H. Euler and B. Kockel, Naturwissenschaften 23, 246 (1935).

[26] R. Karplus and M. Neuman, Phys. Rev. 83, 776 (1951).

[27] V. Costantini, B. De Tollis, and G. Pistoni, Nuovo Cimento A2, 733 (1971).

[28] D. A. Dicus, C. Kao, and W. W. Repko, Phys. Rev. D 57, 2443 (1998).

[29] G. Passarino and M. J. G. Veltman, Nucl. Phys. B160, 151 (1979).

[30] H. H. Patel, Comput. Phys. Commun. 197, 276 (2015).

[31] A. Czarnecki and S. G. Karshenboim, in Proceedings, 14th International Workshop, QFTHEP'99, Moscow, Russia, May 27-June 2, 1999 (1999), pp. 538-544.

[32] See Supplemental Material at http://link.aps.org/ supplemental/10.1103/PhysRevD.97.073005 for the data points we used to generate the black line. it is a list of $\mathrm{G}$ ??? / GEH as a function of $m^{\prime}[\mathrm{MeV}]$.

[33] A. Denner, S. Dittmaier, and L. Hofer, Comput. Phys. Commun. 212, 220 (2017).

[34] J. J. van der Bij and E. W. N. Glover, Nucl. Phys. B313, 237 (1989).

[35] M. Pospelov, Phys. Rev. D 80, 095002 (2009).

[36] R. Essig, E. Kuflik, S. D. McDermott, T. Volansky, and K. M. Zurek, J. High Energy Phys. 11 (2013) 193.

[37] A. Fradette, M. Pospelov, J. Pradler, and A. Ritz, Phys. Rev. D 90, 035022 (2014).

[38] G. Steigman, Phys. Rev. D 87, 103517 (2013).

[39] C. Boehm, M. J. Dolan, and C. McCabe, J. Cosmol. Astropart. Phys. 08 (2013) 041.

[40] R. Essig, J. Mardon, M. Papucci, T. Volansky, and Y.-M. Zhong, J. High Energy Phys. 11 (2013) 167.

[41] W. G. J. Langeveld (mQ Collaboration), in Proceedings, 23rd Summer Institute on Particle Physics, Stanford, USA, 1995 (1995), pp. 479-493.

[42] R. Essig and Z. Vallari (to be published).

[43] M. Battaglieri et al. (BDX Collaboration), arXiv:1406.3028.

[44] https://confluence.slac.stanford.edu/display/MME/Light+ Dark+Matter+Experiment.

[45] S. Gardner (private communication).

[46] H. Park, Phys. Rev. Lett. 119, 081801 (2017).

[47] M. Pospelov and Y.-D. Tsai, arXiv:1706.00424. 